\title{
La vie de l'association de soutien Amis et Correspondants du CIBP
}

Thérèse Goyet

\section{CpenEdition}

\section{Journals}

Édition électronique

URL : http://journals.openedition.org/ccibp/413

DOI : $10.4000 /$ ccibp. 413

ISSN : 2493-7460

Éditeur

Centre international Blaise Pascal

Édition imprimée

Date de publication : 15 avril 1981

Pagination : 16-17

ISSN : 0249-6674

\section{Référence électronique}

Thérèse Goyet, "La vie de l'association de soutien Amis et Correspondants du CIBP », Courrier du Centre international Blaise Pascal [En ligne], 3 | 1981, mis en ligne le 24 novembre 2015, consulté le 19 avril 2019. URL : http://journals.openedition.org/ccibp/413 ; DOI : 10.4000/ccibp.413

Ce document a été généré automatiquement le 19 avril 2019

Centre international Blaise Pascal 


\title{
La vie de l'association de soutien Amis et Correspondants du CIBP
}

\author{
Thérèse Goyet
}

1 Comme le veut l'article 12 des statuts (publiés dans le Courrier $n^{\circ} 2$ ) l'assemblée générale annuelle s'est tenue : pour la première fois, le vendredi 4 décembre 1981, à 15 heures, à la Bibliothèque Municipale et Interuniversitaire, dans la salle Massillon.

2 La secrétaire, $\mathrm{M}^{\text {lle }}$ Marie-Thérèse Sart, a fait le point sur les démarches de subsistance. La trésorière, $\mathrm{M}^{\mathrm{lle}}$ Dominique Seguin, constate que d'après le nombre des cotisants, les finances sont saines. La présidente, $\mathrm{M}^{\mathrm{me}}$ Thérèse Goyet, fait le «bilan d'une première enfance ".

3 Si la gestation du CIBP a été relativement longue : ébranlement en 1976, à l'occasion du colloque Méthodes chez Pascal; Courrier d'enquête 1979 ; rencontre décisive entre Mairie et Président de l'Université, 28 octobre 1980 ; signature de la convention, 23 novembre 1980 ; premier conseil de gestion, 5 décembre 1980 ; l'association de soutien, elle, a pris le départ avec une rapidité remarquable. Les statuts, élaborés en décembre, ont paru au J.O. du 29-30 décembre 1980.

4 Pour leur premier acte les Amis et Correspondants du CIBP ont frappé un grand coup en organisant les Journées de Port-Royal, 6-9 octobre 1981.

5 Le thème « auvergnat » du colloque : Jeux grandes figures d'Auvergne: Gilberte et Jacqueline Pascal, choisi par les Amis de Port-Royal, a entraîné le thème du frère non moins auvergnat pour l'exposition, qui devait être la façade publique des Journées d'étude. Les Amis de Port Royal rayonnant à partir de Paris, le public potentiel de Clermont et d'Auvergne a été peu prospecté. Les célébrations de Pascal en ce pays ont beaucoup changé d'allure. En 1880, 1923, 1962, c'étaient des affaires d'État, avec grand remuement local, et subventions énormes. En 1976 et 1981, l'initiative est prise par quelques universitaires, auxquels les autorités accordent leur estime, et quelques subventions. Il est vrai qu'il ne s'agissait pas d'anniversaires, ni d'inauguration, mais d'étude. Le colloque s'est d'ailleurs auto-financé. Toutes les dépenses ont été faites pour l'exposition dont les Amis du CIBP ont géré le budget. 
La décision de faire cette exposition n'a pu être prise qu'après l'installation du nouveau Conservateur des Musées d'Art, M. Gérard Tisserand. Les réunions du comité ont eu lieu les 19 mars, 9 avril, 14 mai et 29 juin 1981. Le catalogue a été imprimé pendant les vacances. Mais à partir d'un certain moment, impossible à déterminer, le succès comme acquis s'est emporté lui-même.

7 Prêts consentis par la Bibliothèque Nationale, par le Conservatoire National des Arts et Métiers, obtention de la machine de Dresde. L'inauguration, le 6 octobre, par M. Quilliot, Ministre et Maire de Clermont-Ferrand, a été fort belle, et l'exposition a connu pendant un mois un franc succès auprès du public scolaire et adulte (plus de 4000 visiteurs).

La presse régionale, écrite et audiovisuelle, a bien fait son métier d'information, mais dans notre temps de sur-occupation des loisirs, il faudrait beaucoup plus de publicité pour provoquer un ébranlement de la presse parisienne.

Bref les actions marquantes de l'année 1981 demandent une prolongation active et ingénieuse. « Il faut plus qu'une bataille gagnée pour gagner la guerre. »

\section{Le nouveau conseil de l'Association}

Le premier constitué, étant provisoire, cesse d'office ses fonctions avec la première assemblée générale.

Sont élus ou réélus :

\section{René COMBE}

M. Dominique DESCOTES

M. Philippe GAUTIER DE BREUVANT

$\mathrm{M}^{\text {lle }}$ Suzanne MONTAGNE

M. GuY MOURLEVAT

$\mathrm{M}^{\text {lle }}$ Dominique SEGUIN

M. Philippe SELLIER

Sont membres de droit : $\mathrm{M}^{\text {me }}$ Thérèse GOYET, Directeur scientifique du CIBP ; $\mathrm{M}^{\text {lle }}$ MarieThérèse SART, Conservateur en chef de la BMIU.

Le Conseil élit aussitôt le Bureau :

Président : $\mathrm{M}^{\text {me }}$ GOYET

Vice-présidents : M. GAUTIER DE BREUVANT

M. SELLIER

Secrétaire : $\mathrm{M}^{\text {lle }}$ SART

Trésorier : M : DESCOTES

Trésorier-adjoint : $\mathrm{M}^{\text {lle }}$ SEGUIN

\section{Publications en dépôt au CIBP}

Il reste quelques exemplaires du Courrier numéros 1 et 2 .

Vous pouvez demander :

- Le catalogue de l'exposition Blaise Pascal « auvergnat ». $40 \mathrm{~F}+$ port 8,50 F.

- Quatre cartes postales reproduisant les filigranes du papier utilisé par Pascal. $10 \mathrm{~F}+$ port 2,30 F. 
- Dès la parution, les Actes du Colloque Gilberte et Jacqueline Pascal.

\section{BIBLIOGRAPHIE}

\section{Liste des dons reçus par le CIBP en 1981 et au cours du $1^{\text {er }}$ semestre 1982}

\section{Livres et articles}

ADAM Michel. « La signification du miracle dans la pensée de Pascal ». Paris : Revue philosophique, $4,1981.23 \mathrm{p}$.

ARNAUD Jeanne Catherine Agnès. Lettres de la mère Agnès Arnaud, abbesse de Port-Royal. Paris : B. Duprat, 1858. 2 vol. , 528, 542 p.

CAHNÉ Pierre Alain Pascal ou le risque de l'espérance. Paris : Fayard-Mame, 1981. 173 p.

COULET Henri. Pascal écrivant. Aix-en-Provence : Annales de la Faculté des lettres, XXXVII, s.d. 10 p. DESCOTES Dominique. «Fonction argumentative de la satire dans les Provinciales. » in : L'Esprit de la Satire. Paris : Place. 23 p.

DORDAIN M. et DAILLY R. Maladie et mort de Blaise Pascal : hypothèses nouvelles. Rouen : lmpr., Lecerf, 1978. $15 \mathrm{p}$.

GOYET Thérèse. « Le tragique et l'espérance chez les classiques chrétiens ». Paris : L'information littéraire, mars-avril $1981.6 \mathrm{p}$.

MAEDA Yoïchi. [Transcription fidèle des pp. 347 à 360 du manuscrit des Pensées]. s.l. : s.n. s. d. $26 \mathrm{p}$.

Mélanges Lucien Ceyssens. Jansénius et le jansénisme aux Pays-Bas. Louvain : Peeters, 1982. 247 p.

PASCAL Blaise. [Guvres complètes]. Trad. en japonais par T. Tanabé. Tokyo : Kyo-bunkwan, 1980 - 2 vol. , 282, 259 p. (tomes 3 et 4).

PASCAL Blaise. Pensées (suivies de l'éd. originale du Discours de Filleau de La Chaise). $2^{\mathrm{e}}$ éd. Paris : G. Desprez, 1670. 348-214 p.

PASCAL Blaise. Pensées, avec les notes de Voltaire. Londres : s. n., 1785. 2 vol. , 240, 208 p.

PASCAL Blaise. Pensées, Paris : J. de Bonnot, 1979. 278 p.

PASCAL Blaise. Les Provinciales. $10^{e}$ éd. augm. de la Lettre d'un avocat au Parlement. Cologne : N. Schoute, $1697.351 \mathrm{p}$.

PASCAL Blaise. Lettres écrites à un provincial. Paris : Emler, 1829. $472 \mathrm{p}$.

PLAINEMAISON Jacques. « Blaise Pascal et la grâce suffisante des thomistes. » Paris : Revue thomiste, oct.-déc. $1981.11 \mathrm{p}$. 
PLAINEMAISON Jacques. «Blaise Pascal et le sacrement de pénitence. » Florence : L. S. Olschki, 1980. 9 p.

[REMACLE Albert de et FRIC (R.)]. Blaise Pascal Quelques souvenirs sur lui et les siens. Clermont-Fd : lmpr. Vallier, 1923. 40 p. XIV f. de pl.

SELLIER Philippe. La théorie morale chez Pascal. Seattle (Washington): Papers on French seventeenth century literature, VIII , 14-2, 1981. $25 \mathrm{p}$.

SHIOKAWA Tetsuya. «La connaissance par l'autorité selon Pascal. » Tokyo : Société japonaise de langue et de littérature françaises, 1977. 14 p.

SHIOKAWA Tetsuya. [« Figures et symboles : la conception pascalienne des figures »]. Tokyo : Iwanami, 1977. 17 p. (ouvrage en langue japonaise).

SHIOKAWA Tetsuya. Pascal et les miracles. Préf. de Jean Mesnard. Paris : A.-G. Nizet, 1977.-220 p. WlLLAERT Léopold. Bibliotheca janseniana belgica. Namur : Faculté de philosophie, 1949-1951. 3 vol. , $1191 \mathrm{p}$.

\section{Documents divers}

CENTRE REGIONAL DE DOCUMENTATION PEDAGOGIQUE. Clermont. -Jeu de 24 photos représentant les neuf machines arithmétiques connues de Pascal. Clermont-Fd : C.R.D.P., 1981. CENTRE REGIONAL DE DOCUMENTATION PEDAGOGIQUE. Clermont. La machine arithmétique ou Pascaline. Clermont-Fd : C.R.D.P., 1981. 12 p. 12 pl. de photos.

Exposition, Clermont-Ferrand. Bibliothèque universitaire de médecine. Pascal et ses médecins. Clermont-Fd : B.U. Médecine, 1974. 10 panneaux.

PASCAL Blaise. Lettre dédicatoire à Monseigneur le Chancelier. s. 1. : s. n., s. d. 1 microfilm.

Usage de la machine : transcription littérale (par Henry Malet-Daubant] d'un manuscrit du $18^{\mathrm{e}}$ siècle annoté par Bossut et acquis par la Bibliothèque municipale de Clermont-Ferrand. 1 classeur.

INDEX

Mots-clés : Pascal, CIBP

\section{AUTEUR}

\section{THÉRÈSE GOYET}

Professeur à l'Université II de Clermont-Ferrand 